\title{
Krankheitsrepräsentationen bei nicht-kardialem Brustschmerz - eine verhaltensmedizinische Kurzintervention
}

\author{
Stefanie Schroeder ${ }^{\mathrm{a}}$ Stephanie Schmid ${ }^{\mathrm{a}} \quad$ Alexandra Martin $^{\mathrm{b}}$ \\ ${ }^{\text {a }}$ Psychosomatische und Psychotherapeutische Abteilung: Psychotherapieforschung, \\ Friedrich-Alexander-Universität Erlangen-Nürnberg, Universitätsklinikum Erlangen, \\ ${ }^{\mathrm{b}}$ Abteilung für Klinische Psychologie und Psychotherapie, Bergische Universität Wuppertal, Deutschland
}

\section{Schlüsselwörter}

Nicht-kardialer Brustschmerz · Subjektive Krankheitsrepräsentationen · Intervention · Verhaltensmedizin

\section{Zusammenfassung}

Hintergrund: Nicht-kardiale Brustschmerzen sind häufig und entwickeln oft einen chronischen Verlauf. Die einfache ärztliche Rückmeldung über unauffällige kardiodiagnostische Befunde hat sich als nicht ausreichend erwiesen, um dysfunktionale Krankheitsrepräsentationen, Ängste und Sorgen der Betroffenen langfristig zu reduzieren. Daher war das Ziel der Studie, eine niederschwellige, auf subjektive Krankheitsrepräsentationen ausgerichtete psychologische Intervention zu entwickeln und hinsichtlich ihrer Anwendbarkeit zu evaluieren. Patienten und Methoden: 57 Patienten mit nicht-kardialem Brustschmerz nahmen randomisiert an unserer Intervention teil und wurden zu Brustschmerzen und subjektiven Krankheitsrepräsentationen sowie zur subjektiven Bewertung der Intervention befragt. Ergebnisse: Die Intervention wurde durch die meisten Teilnehmer sehr positiv eingeschätzt («Erfolg versprechend», "weiterzuempfehlen»). Jedoch lehnten vorab $42 \%$ der potenziell geeigneten Patienten eine Teilnahme ab. Die Interventionsgruppe war im Gegensatz zu den Refusern jünger, berichtete ungünstige Krankheitsrepräsentationen und größere Brustschmerzintensität. Schlussfolgerung: Es bleibt eine Herausforderung, Patienten mit nicht-kardialem Brustschmerz mittels verhaltensmedizinischer Interventionen frühzeitig zu erreichen, insbesondere akut weniger beeinträchtigte Personen. Demgegenüber zeigen unsere Ergebnisse, dass eine niederschwellige Intervention realisierbar ist und überwiegend als wirksam eingeschätzt wird.

\section{Keywords}

Noncardiac Chest Pain . Subjective illness representations · Intervention · Behavioral medicine

\section{Summary}

IIIness Perceptions in Noncardiac Chest Pain -

\section{A Brief Psychological Intervention}

Background: Noncardiac chest pain is a frequent condition that is likely to develop a chronic course. Simply reassuring patients about nonpathological diagnostic test results has proved to be insufficient to effectively reduce dysfunctional illness representations and worry. The aim of the study was to develop a brief psychological intervention focussing on subjective illness representations and to evaluate its practicability. Patients and Methods: 57 patients with noncardiac chest pain participated. IIIness representations and chest pain characteristics as well as the participants' subjective evaluation of the intervention were assessed. Results: Subjective ratings by the participants were mostly positive, but of all patients eligible for the intervention $42 \%$ declined to participate. In comparison to the refusers, the intervention participants were younger and reported stronger dysfunctional illness representations and higher pain intensity. Conclusion: It remains a challenge to affect patients with noncardiac chest pain with early psychological interventions, in particular patients who feel only mildly impaired. Nevertheless, our results show that a low-threshold intervention is applicable and is mainly seen as effective.

\section{KARGER \\ Fax +497614520714}

Information@Karger.com

www.karger.com (c) 2013 S. Karger GmbH, Freiburg

$1016-6262 / 13 / 0234-0234 \$ 38.00 / 0$

Accessible online at:

www.karger.com/ver
Dipl.-Psych. Stefanie Schroeder

Psychosomatische und Psychotherapeutische Abteilung: Psychotherapieforschung Friedrich-Alexander-Universität Erlangen-Nürnberg

Universitätsklinikum Erlangen

Schwabachanlage 6, 91054 Erlangen, Deutschland

stefanie.schroeder@uk-erlangen.de 


\section{Einleitung}

Missempfindungen im Brustbereich gehören zu den häufigsten Beschwerden, die in der medizinischen Versorgung berichtet werden [Kroenke et al., 1990; Schappert, 1992]. Die beeinträchtigenden Empfindungen variieren dabei sehr; so werden unter anderem Schluckstörungen, Palpitationen, Engegefühl sowie Atemstörungen berichtet. Eine weitere häufig auftretende Missempfindung im Brustraum ist Brustschmerz. Bis $\mathrm{zu} 25 \%$ der Allgemeinbevölkerung geben an, bereits Brustschmerzen erlebt zu haben [Kroenke und Price, 1993]. Die Betroffenen berichten verschiedenste schmerzhafte Missempfindungen im Brustbereich (unter anderem Stechen, Brennen, Druck, Ziehen), die von variierender Intensität und Dauer sein können.

Sowohl Brustschmerzen als auch verschiedene andere Missempfindungen können generell auf pathologischen somatischen Veränderungen beruhen (Tab. 1). Speziell Brustschmerzen führen aufgrund ihres Signalcharakters für ein möglicherweise vorliegendes ernsthaftes kardiales Problem (z.B. Herzinfarkt) schnell zu Sorgen und folglich zu einem Bedürfnis, die Beschwerden abzuklären [Kahn, 2000]. Bei bis zu $66 \%$ der Betroffenen kann eine eindeutige somatische Pathologie jedoch nicht bestätigt werden [Glombiewski et al., 2010]; man spricht dann von unspezifischen oder funktionellen Brustschmerzen. Wenn sich die Diagnostik speziell auf den Ausschluss kardialer Erkrankungen bezieht, spricht man dagegen von «nicht-kardialem Brustschmerz». Bei bis zu 50\% der Patienten mit Brustschmerzen kann keine zugrundeliegende kardiale Erkrankung festgestellt werden [Zimmermann et al., 2010], wenngleich einige spezifische kardiologische Pro- bleme schwer zu objektivieren sind. Auch wenn Brustschmerzen nur einen Aspekt möglicher Missempfindungen im Brustbereich darstellen, ist der Fokus dieser Arbeit im weiteren Verlauf diesbezüglich ausgerichtet.

Trotz Ausschluss kardiologisch bzw. somatisch relevanter Ursachen bleiben sowohl die Brustschmerzen als auch beschwerdebedingte Beeinträchtigungen (z.B. Angst, Sorgen, Schonverhalten, wiederholte Rückversicherung) bei einem Großteil der Patienten langfristig bestehen [Potts und Bass, 1993], während die kardiale Prognose insgesamt sehr günstig ist [Papanicolaou et al., 1986]. Sowohl bezüglich der Entstehung sowie der Persistenz nicht-kardialer Brustschmerzen geht man von einem multifaktoriellen Bedingungsgefüge mit verschiedenen psychologischen und physiologischen Einflussfaktoren aus [Mayou, 1989]. Physiologische Einflussfaktoren können unter anderem pulmonale (z.B. respiratorischer Infekt), muskuloskelettale (z.B. Verspannungen in der Brustwand) und gastroenterologische (z.B. Reflux oder Mobilitätsstörungen des Ösophagus) Aspekte sein [DEGAM, 2011; Eslick und Fass, 2003]. Als psychologische Einflussfaktoren werden verschiedene kognitiv-perzeptuelle, emotionale und verhaltensmäßige Faktoren genannt, die sich auf die Wahrnehmung von Brustschmerzen auswirken [Bass und Mayou, 2002]. So kann eine pathologische Verarbeitung der Beschwerden den Verlauf maßgeblich beeinflussen (Tab. 1). Überträgt man beispielsweise das Selbstregulationsmodell von Leventhal et al. [1998] auf Personen mit nicht-kardialem Brustschmerz, muss man davon ausgehen, dass das Erleben der Brustschmerzen bei den Betroffenen verschiedene kognitive Repräsentationen zu Art («identity»), Ursachen («causes») und Folgen («consequences») der Symptome sowie Er-

Tab. 1. Übersicht über Auslösebedingungen thorakaler Schmerzen und Kriterien der pathologischen Verarbeitung von Brustschmerzen in Anlehnung an Linden et al. [2008], DEGAM [2011] und Schaefert et al. [2012]

\begin{tabular}{|c|c|}
\hline Auslösebedingungen thorakaler Schmerzen & Kriterien einer pathologischen Verarbeitung von Brustschmerzen \\
\hline Chronische koronare Herzerkrankung & erhöhte Ausrichtung der Aufmerksamkeit auf das Herz \\
\hline Akutes Koronarsyndrom, Klappenvitien, Arrhythmien & katastrophisierende Kognitionen \\
\hline Koronarspasmen, Syndrom X & dysfunktionale Gesundheits-/Krankheitsannahmen \\
\hline Pleuraerkrankungen & Gedanken der Hilf- und Hoffnungslosigkeit \\
\hline Hypertonie & ausgeprägtes, anhaltendes Rückversicherungsverhalten \\
\hline Brustwandsyndrom (neuromuskuloskelettale Erkrankungen) & häufiges Einfordern ärztlicher Untersuchungen \\
\hline Veränderungen der Brustwirbelsäule & häufiger Behandlerwechsel \\
\hline Interkostalneuralgie & ausgeprägte Schon- und Vermeidungshaltung \\
\hline Refluxerkrankung & deutlich reduzierte Funktionsfähigkeit \\
\hline Ösophageale Motilitätsstörungen, viszerale Hypersensitivität & sozialer Rückzug \\
\hline Benigne Erkrankungen des Verdauungstraktes & psychische Komorbidität \\
\hline Gastritis, Zwerchfellhernie & hohe psychosoziale Belastung \\
\hline \multicolumn{2}{|l|}{ Infekt der oberen Atemwege, Pneumonie, Lungenembolie } \\
\hline \multicolumn{2}{|l|}{ Angststörung, somatoforme Störung, depressive Erkrankung } \\
\hline \multicolumn{2}{|l|}{ Vegetatives Erregungssyndrom, stressbedingte Kardiomyopathie } \\
\hline \multicolumn{2}{|l|}{ Malignome im Brustraum } \\
\hline Medikamenteninduzierter Brustschmerz & \\
\hline
\end{tabular}


wartungen bezüglich des Verlaufs der Symptome über die Zeit («time-line») und ihrer Beeinflussbarkeit («control») durch ärztliche Behandlung und eigene Verhaltensweisen hervorruft. Bei Patienten mit nicht-kardialen Brustschmerzen bestehen bereits zum Zeitpunkt der diagnostischen Abklärung ungünstige Krankheitsrepräsentationen bezüglich der empfundenen Kontrollierbarkeit und des Verständnisses der Schmerzen. Diese schwächen die subjektiv erlebte Rückversichertheit nach Mitteilung unauffälliger kardialer Befunde deutlich ab und beeinflussen so den Verlauf der Beschwerden sowie assoziierte psychosoziale Beeinträchtigungen ungünstig [Donkin et al., 2006; Jonsbu et al., 2012; Robertson et al., 2008]. Die einfache ärztliche Rückmeldung über unauffällige Untersuchungsbefunde reicht dann häufig nicht aus, um die Sorgen und Ängste der Patienten langfristig zu beseitigen [Jonsbu et al., 2012; Robertson et al., 2008]. Um einer Chronifizierung der Beschwerden effektiv entgegenzuwirken, wurden daher psychologische Interventionen vorgeschlagen, die die subjektiven Krankheitsannahmen der Betroffenen, deren Sorgen und Ängste explizit berücksichtigen sowie ein alternatives Erklärungsmodell anbieten [Mayou und Farmer, 2002]. Empfohlen wird hierzu ein Vorgehen entsprechend einer gestuften Versorgung [Mayou et al., 1999], in deren Rahmen bereits bei früher Beschwerdemanifestation spezifische Informationen und Interventionen angeboten werden [Krille und Martin, 2011].

Empirische Befunde bei verschiedenen somatoformen Beschwerden zeigen für niederschwellige sogenannte One-Session-Treatments Erfolge hinsichtlich der Beschwerdeintensität, internaler Kontrollüberzeugungen in die eigene Beschwerdebewältigung und beschwerdebedingter Beeinträchtigungen im Alltag [Martin et al., 2007; Schmulson et al., 2006]. Bei nichtkardialem Brustschmerz sind niederschwellige Maßnahmen bislang selten untersucht worden. Erste Befunde sprechen für eine positive Wirkung kognitiv-behavioraler Elemente [Esler et al., 2003], wenngleich die Befundlage insgesamt uneinheitlich ist [Sanders et al., 1997].

Daher wurde im Rahmen der hier vorgestellten Studie in Anlehnung an Martin et al. [2007] und Esler et al. [2003] eine kurze verhaltensmedizinische Intervention für Patienten mit Brustschmerz entwickelt, bei denen kurz zuvor eine relevante Kardiopathologie diagnostisch ausgeschlossen wurde. Aufgrund der Relevanz subjektiver Krankheitsrepräsentationen für die Beschwerdechronifizierung liegt der Schwerpunkt der Kurzintervention auf der Erweiterung der spezifischen Kausalattributionen der Patienten in Richtung eines multifaktoriellen biopsychosozialen Verständnisses der Brustschmerzen sowie der Kontrollerwartungen durch Vermittlung von Bewältigungsstrategien.

Wenngleich sich diese Intervention an Personen mit kardiologisch nicht erklärtem Brustschmerz richtet, darf dennoch nicht außer Acht gelassen werden, dass eine übermäßige Beschäftigung und hypochondrische Verarbeitung von Beschwerden (z.B. im Sinne des Konzeptes der «Pathologischen
Realangst» [Linden et al., 2008]) unabhängig ist von der Objektivierbarkeit einer den Beschwerden zugrundeliegenden organischen Ursache. Somit können auch Personen mit bestätigter kardialer Erkrankung sowie Personen, die verschiedene andere Missempfindungen im Brustbereich berichten, eine problematische Verarbeitung der Beschwerden aufweisen und mutmaßlich von einer ähnlichen Intervention profitieren.

Die vorliegende Arbeit fokussiert auf die Darstellung des Interventionskonzeptes. Zusätzlich werden einzelne Faktoren der Anwendbarkeit der Intervention geprüft. Dazu werden die subjektiven Bewertungen der Intervention durch die Teilnehmer sowie der Anteil der Personen, die eine Teilnahme ablehnten (Refuser), analysiert. Frühere Erhebungen legen nahe, dass nur ein Teil der betroffenen Zielgruppe mit psychologischen Interventionen gut erreichbar ist [Sanders et al., 1997]. Daher werden die direkt geäußerten Gründe der Ablehnung als Indikatoren der Anwendbarkeit berichtet. Zusätzlich werden Teilnehmende versus Refuser hinsichtlich psychologischer und Schmerzcharakteristika verglichen.

\section{Darstellung des Interventionsleitfadens}

\section{Aufbau und Konzept der Kurzintervention}

Im Sinne des gestuften Versorgungsansatzes richtet sich die hier vorgestellte Kurzintervention an Patienten mit nichtkardialem Brustschmerz unmittelbar nach Mitteilung unauffälliger kardiologischer Untersuchungsbefunde. Es handelt sich um ein einmaliges Gespräch im Einzelsetting von maximal 60 min Dauer. Die Interventionsinhalte sind in Form von flexibel anwendbaren Themen-Modulen in einem Manual vorgegeben (das Manual zur Intervention ist bei den Autorinnen auf Nachfrage erhältlich), sodass eine individuelle Anpassung der Gesprächsinhalte an die subjektiven Krankheitsrepräsentationen und Erfahrungen des Patienten erfolgen kann. Durch das Erfragen von individuellen Erfahrungen, Einstellungen und Problembereichen wird zunächst geklärt, inwieweit ein jeweiliges Interventionsmodul für die individuelle Situation des Teilnehmers relevant ist. Dementsprechend werden Vertiefungen der Modulinhalte individuell adaptiert, wobei wenigstens die Kernbotschaft eines jeden Moduls vermittelt wird. Zum Einsatz kommen insbesondere psychoedukative Elemente (Vermittlung von Erklärungsmodellen anhand individueller Erfahrungen des Patienten, Vermittlung von medizinischen Informationen, Vorstellen von Methoden zum Umgang mit persistierenden Brustschmerzen) sowie Methoden der kognitiven Umstrukturierung. Die Inhalte werden in Zusammenarbeit mit dem Patienten anhand von Arbeitsblättern erarbeitet, welche der Patient als Informations- und Selbsthilfematerial erhält.

Kernmodul «Alternative Ursachen der Brustschmerzen»: Zielsetzung dieses Moduls ist (1) eine Entkatastrophisierung der Brustschmerzen sowie (2) eine Erweiterung der subjektiven Krankheitsrepräsentationen und somit eine Erweiterung 
des Verständnisses der Beschwerden. Zunächst werden die subjektiv bestehenden Repräsentationen der Brustschmerzen sowie konkret die Überzeugung, an einer ernsthaften Herzerkrankung zu leiden, erfragt ( «Wie sehr sind Sie beruhigt durch die kardiologische Untersuchung?» «Inwieweit belasten Sie Ihre Brustschmerzen?» «Welche Ursachen vermuten Sie?»). In einem weiteren Schritt erfolgt dann eine Vermittlung von Informationen zu häufigen Einflussfaktoren auf Brustschmerzen (z.B. Verspannungen, Atemtechnik, Refluxbeschwerden, eingeklemmte Nerven, Tab. 1). Der Fokus liegt dabei auf der Verdeutlichung einer multifaktoriellen Beeinflussung der erlebten Beschwerden durch verschiedene zumeist gutartige somatische Bedingungen. Vorbereitend auf die folgenden Module werden auch mögliche Einflüsse psychologischer Faktoren diskutiert («Könnten Sie sich vorstellen, inwiefern auch psychologische Prozesse einen zusätzlichen Einfluss auf Ihre Beschwerden haben könnten?»).

Wahlmodul «Stress und Entspannung»: Dieses Modul soll dem Patienten in erster Linie den möglichen Zusammenhang zwischen An- bzw. Entspannung und den Brustschmerzen anhand eines individuellen Bedingungsmodells vermitteln. Der Patient soll zwischen Stressoren und Stressreaktion unterscheiden können. Erfragt wird zunächst eine individuelle Stresssituation, an die sich der Patient gut erinnern kann. Anschließend wird erfragt, wie sich die entsprechende Stressreaktion auf den Ebenen Physiologie, Kognitionen und Emotionen darstellte. Anhand dieser individuellen Erfahrungen des Patienten wird dann vermittelt, wie auch psychologische Prozesse wie beispielsweise individuelle Bewertungen und Erwartungen Auswirkungen auf den Körper und somit auch auf Brustschmerzen haben könnten (im Sinne eines hypothetischen alternativen Erklärungsmodells). In einem weiteren Teil werden dem Patienten Strategien vermittelt, mit der Stressreaktion und den gegebenenfalls assoziierten Brustschmerzen umzugehen bzw. diese zu reduzieren. Dafür werden verschiedene Entspannungsverfahren (Progressive Muskelentspannung, Bauchatmung, Wahrnehmungsspaziergang) kurz vorgestellt; schriftliche Anleitungen befinden sich zusätzlich in den Materialien. Individuelle Erfahrungen werden erfragt, und bei Interesse wird eine kurze Übung zur Entspannung mittels Atembeobachtung (5 min) angeleitet.

Wahlmodul «Sorgen»: Berichtet der Patient trotz Ausschluss gravierender kardialer Erkrankungen weiterhin anhaltende und belastende Sorgen bezüglich ernsthafter kardialer Ursachen, so werden der Einfluss erlebter Sorgen auf die körperliche Symptomatik sowie Möglichkeiten zum Umgang mit persistierenden Sorgen besprochen. Dazu werden zunächst sorgenvolle Gedanken bezüglich der Brustschmerzen erfragt («Welche Sorgen machen Sie sich?» «Wie gehen Sie mit solchen Gedanken um?»). Stellen sich die anhaltenden Sorgen als eigenständiges Problem dar, werden dem Patienten anhand eines Arbeitsblattes Möglichkeiten zur Sorgenbewältigung vorgestellt (Ablenkung durch geistige oder körperliche Aktivität, Hinterfragen und Umformulieren der sorgenvollen
Gedanken in hilfreichere Gedanken) und diskutiert («Können Sie sich vorstellen, dass das für Ihre Sorgen hilfreich sein kann?»).

Wahlmodul «Aktivität und Ausgleich»: Der inhaltliche Schwerpunkt dieses Moduls liegt auf der Vermittlung der langfristig ungünstigen Folgen ausgeprägten Schonverhaltens sowie unausgewogener Aktivität. Anhand eines «Teufelskreis-Schemas» (in Anlehnung an Rief und Hiller [1998]) wird gemeinsam mit dem Patienten erarbeitet, inwiefern übermäßiges Schonverhalten ungünstige Effekte auf die wahrgenommenen Beschwerden haben kann und eine Aufrechterhaltung körperlicher Aktivität wichtig ist. Berichtet der Patient eine Einschränkung körperlicher Aktivität, wird mit ihm eine graduelle Steigerung des Aktivitätsniveaus geplant; eine entsprechende Planungsvorlage befindet sich in den Materialien. Der Patient wird dazu angehalten zu überlegen, in welcher Art er den «Teufelskreis» durchbrechen könnte. Gleichzeitig wird mit einem Verweis auf das Modul «Stress und Entspannung» darauf hingewiesen, dass auch zu viel Aktivität ungünstig sein kann.

Kernmodul «Übertragung der Interventionsinhalte in den Alltag»: Abschließend erfolgt gemeinsam mit dem Patienten eine zusammenfassende Sichtung der Interventionsinhalte. Der Patient wird motiviert, eine oder mehrere der besprochenen Bewältigungsstrategien zur Umsetzung im Alltag auszuwählen («Was von den Dingen, die wir heute besprochen haben, glauben Sie, würde am besten gegen Ihre Beschwerden helfen?» «Was möchten Sie gerne mal ausprobieren?»). Die ausgewählte Strategie wird dann auf einem Arbeitsblatt möglichst konkret festgehalten (z.B. «1 Mal pro Woche Atementspannung»).

\section{Anwendbarkeit der Intervention - Methodik der Analysen}

\section{Ablauf}

Die Rekrutierung von Teilnehmern erfolgte an der Medizinischen Klink 2 (Angiologie und Kardiologie) des Universitätsklinikums Erlangen sowie an der Medizinischen Klinik 1 des Krankenhauses Martha-Maria Nürnberg. Die Teilnahme an der Intervention erfolgte im Rahmen einer Evaluationsstudie, weswegen folgende Einschlusskriterien vorausgesetzt wurden: (1) das Vorliegen von subjektiv belastenden Brustschmerzen, (2) unauffällige Befunde in der vor Ort durchgeführten Koronardiagnostik (maximale Einengung der Koronargefäße von <50\%) und (3) Alter 18-75 Jahre. Ausgeschlossen wurden Patienten, die (1) sich aufgrund der Brustschmerzen in Psychotherapie befanden, (2) keine ausreichenden kognitiven Fähigkeiten und Sprachkenntnisse für eine Studienteilnahme aufwiesen oder (3) bei denen eine relevante kardiale Erkrankung vorbekannt war oder neu diagnostiziert wurde. Geeignete Patienten wurden unmittelbar nach der ärztlichen Rückmeldung unauffälliger Befunde zunächst über Ziele und Ablauf der Studienteilnahme aufgeklärt sowie über eine Aufwandsentschädigung in Höhe von 30 EUR unterrichtet. Die Intervention erfolgte zeitnah $(15$ Tage $\pm 19,4)$ in individueller Abstimmung mit den Teilnehmern. Interventionsleiter waren eine Psychologische Psychotherapeutin (Verhaltenstherapie), eine Diplom-Psychologin in psychotherapeutischer Ausbildung (Verhaltenstherapie) oder ein Arzt in kardiologischer Facharztausbildung. Die Interventionsleiter wurden entsprechend 
Tab. 2. Vergleich von Interventionsteilnehmern und Refusern

\begin{tabular}{|c|c|c|c|}
\hline Variable & Teilnehmer, $\mathrm{n}=57(\%)$ & Refuser, $\mathrm{n}=103(\%)$ & Testwerte* (df) \\
\hline \multicolumn{4}{|l|}{ Soziodemografie } \\
\hline Alter $(\mathrm{M} \pm \mathrm{SD})$ & $52 \pm 10,8$ & $57 \pm 11,1$ & $\mathrm{U}=2442, \mathrm{Z}=-2,5, \mathrm{p}=0,013$ \\
\hline Geschlecht (weiblich) & $29(50,9)$ & $46(44,7)$ & $\chi^{2}(1)=0,6$, n.s. \\
\hline Bildungsstand & & & $\chi^{2}(2)=5,7$, n.s. \\
\hline Kein Abschluss / Hauptschule & $16(29,1)$ & $36(36,7)$ & \\
\hline Realschulabschluss & $21(38,2)$ & $20(20,4)$ & \\
\hline (Fach-)Hochschulreife & $18(32,7)$ & $42(42,9)$ & \\
\hline Koronarstatus & & & $\chi^{2}(1)=9,3, p=0,002$ \\
\hline Glattwandige Gefäße & $37(64,9)$ & $41(39,8)$ & \\
\hline Insignifikante Gefäßablagerungen & $20(35,1)$ & $62(60,2)$ & \\
\hline \multicolumn{4}{|l|}{ Brustschmerzcharakteristika } \\
\hline Mittlere Intensität $(\mathrm{M} \pm \mathrm{SD})$ & $3,6 \pm 1,7$ & $3,3 \pm 2,3$ & $\mathrm{U}=2255, \mathrm{Z}=-1,6$, n.s. \\
\hline Maximale Intensität $(\mathrm{M} \pm \mathrm{SD})$ & $5,7 \pm 2,6$ & $4,6 \pm 2,7$ & $\mathrm{U}=1973, \mathrm{Z}=-2,4, \mathrm{p}=0,018$ \\
\hline Beeinträchtigung $(\mathrm{M} \pm \mathrm{SD})$ & $4,3 \pm 2,5$ & $3,7 \pm 2,6$ & $\mathrm{U}=2296, \mathrm{Z}=-1,5, \mathrm{n} . \mathrm{s}$ \\
\hline Dauer seit Beginn & & & $\chi^{2}(3)=0,92$, n.s. \\
\hline$<3$ Monate & $23(41,1)$ & $48(49,0)$ & \\
\hline bis 6 Monate & $8(14,3)$ & $12(12,2)$ & \\
\hline bis 12 Monate & $7(12,5)$ & $10(10,2)$ & \\
\hline$>12$ Monate & $18(32,1)$ & $28(28,6)$ & \\
\hline Häufigkeiten von Episoden & & & $\chi^{2}(3)=2,2$, n.s. \\
\hline$<$ einmal monatlich & $8(14,6)$ & $12(12,0)$ & \\
\hline Monatlich & $12(21,8)$ & $15(15,0)$ & \\
\hline Wöchentlich & $22(40,0)$ & $50(50,0)$ & \\
\hline Täglich & $13(23,7)$ & $24(24,0)$ & \\
\hline \multicolumn{4}{|c|}{ Subjektive Krankheitsüberzeugungen $(M \pm S D)$} \\
\hline IPQ-B: Konsequenzen & $4,5 \pm 2,3$ & $3,7 \pm 2,4$ & $\mathrm{U}=2253, \mathrm{Z}=-2,3, \mathrm{p}=0,022$ \\
\hline IPQ-B: Zeitverlauf & $3,6 \pm 2,2$ & $3,6 \pm 2,5$ & $\mathrm{U}=2430, \mathrm{Z}=-0,3$, n.s. \\
\hline IPQ-B: persönliche Kontrolle & $3,3 \pm 2,8$ & $3,5 \pm 2,9$ & $\mathrm{U}=2694, \mathrm{Z}=-0,3$, n.s. \\
\hline IPQ-B: Behandlungskontrolle & $6,6 \pm 2,4$ & $7,2 \pm 2,3$ & $\mathrm{U}=2097, \mathrm{Z}=-1,6$, n.s. \\
\hline IPQ-B: Beschwerden & $4,8 \pm 2,3$ & $3,8 \pm 2,3$ & $\mathrm{U}=1966, \mathrm{Z}=-3,0, \mathrm{p}=0,003$ \\
\hline IPQ-B: Sorgen & $6,4 \pm 2,4$ & $5,9 \pm 2,5$ & $\mathrm{U}=2505, \mathrm{Z}=-1,3, \mathrm{n} . \mathrm{s}$ \\
\hline IPQ-B: Verständnis & $4,5 \pm 2,7$ & $4,5 \pm 3,0$ & $\mathrm{U}=2652, \mathrm{Z}=-1,4$, n.s. \\
\hline IPQ-B: emotionale Beeinträchtigung & $6,3 \pm 2,6$ & $5,0 \pm 2,9$ & $\mathrm{U}=2075, \mathrm{Z}=-2,9, \mathrm{p}=0,004$ \\
\hline
\end{tabular}

*Tests waren Mann-Witney-U-Test und $\chi^{2}$-Test. Fallzahlen variieren leicht aufgrund fehlender Daten.

$\mathrm{Z}=$ Prüfgröße; n.s. = nicht signifikant; $\mathrm{M}=$ Mittelwert; $\mathrm{SD}=$ Standardabweichung.

des Interventionsmanuals intensiv geschult und regelmäßig durch eine Psychologische Psychotherapeutin (Verhaltenstherapie) supervidiert. Für die Studie liegt ein positives Ethikvotum vor (Ethikkommission der Friedrich-Alexander-Universität Erlangen-Nürnberg); sie ist registriert im Deutschen Register Klinischer Studien (DRKS00003545).

\section{Messinstrumente}

Noch vor der Koronardiagnostik bearbeiteten die Teilnehmer neben Fragen zu soziodemografischen Aspekten folgende Fragebögen:

Der «Deutsche Schmerzfragebogen» der Deutschen Schmerzgesellschaft [2005] erhebt Schmerzcharakteristika wie Schmerzintensität, subjektive Beeinträchtigung (Ratingskalen 0-10) sowie Dauer seit Beginn und Häufigkeit von Schmerzepisoden. Der «brief illness perception questionnaire» (Kurzversion; IPQ-B) [Broadbent et al., 2006] erfasst in 8 Items subjektive Krankheitsrepräsentationen. Durch Ersetzen des Begriffs «Krankheit» durch «Brustschmerzen» wurde das Instrument an die spezifische Untersuchungsgruppe angepasst. Erfragt wurden Annahmen zu Auswirkungen der Brustschmerzen (Konsequenzen), zum vermuteten zeitlichen Verlauf, zur Kontrollierbarkeit durch eigene und Behandlungsmaßnahmen, zur Wahrnehmung von Beschwerden, zum Verständnis der Brustschmerzen, zu Sorgen sowie zur emotionalen Beeinträchtigung aufgrund der Brustschmerzen. Die Beantwortung erfolgte auf Ratingskalen (0-10). Höhere Werte entsprechen ungünstigeren Krankheitsüberzeugungen. Validierungsstudien zeigen eine gute Test-Retest-Reliabilität und gute Validität des IPQ-B [Broadbent et al., 2006].

Direkt im Anschluss an die Intervention sowie nach 4 Wochen beantworteten die Interventionsteilnehmer Rückmeldebögen zur Zufriedenheit mit der Intervention (adaptiert nach Martin et al. [2007] und Nanke und Rief [2000]). Die Ergebnisse der Koronardiagnostik wurden der medizinischen Dokumentation entnommen.

\section{Stichprobe}

Im Rekrutierungszeitraum von Mai 2010 bis Februar 2012 wurden 258 Patienten identifiziert, die sich aufgrund von Brustschmerzen zur kardio- 


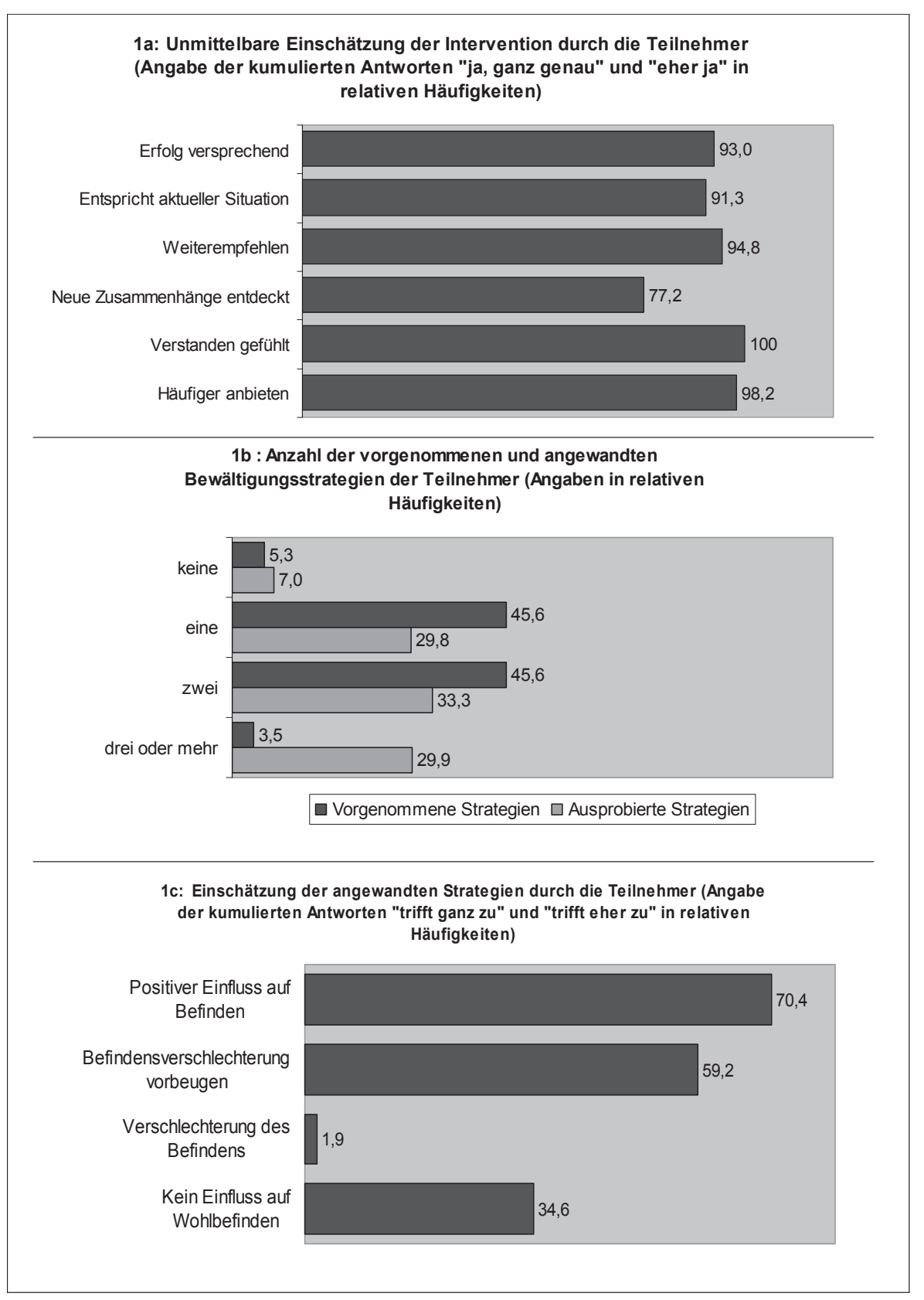

Abb. 1. Ergebnisse der Analysen zur Anwendbarkeit der Intervention.

logischen Diagnostik vorstellten und bei denen eine Untersuchung mittels Herz-Computertomografie oder Koronarangiografie unauffällige Koronarbefunde erbrachte. Bei 10 Patienten waren nicht alle Ein- und Ausschlusskriterien erfüllt. Von 248 zur Studienteilnahme geeigneten Patienten mit nicht-kardialem Brustschmerz stimmten 136 Personen einer randomisierten Studienteilnahme zu, wovon 68 Personen der Interventionsbedingung zugeordnet wurden. Von diesen lehnten 9 Personen erst nach der randomisierten Zuordnung die Teilnahme ab, sodass insgesamt 59 Personen tatsächlich an unserer verhaltensmedizinischen Kurzintervention teilnahmen. Nach Ausschluss zweier Teilnehmer, von denen keine Daten zur 4-Wochen-Katamnese vorlagen, gingen 57 Studienteilnehmer in die Analysen ein. Tabelle 2 zeigt soziodemografische und klinische Charakteristika der Stichprobe.

\section{Statistische Auswertung}

Die statistische Auswertung erfolgte mittels SPSS Statistics 19. Zur Prüfung von Gruppenunterschieden wurden nichtparametrische Verfah- ren angewandt (fehlende Normalverteilung der abhängigen Variablen). Bei signifikanten Unterschieden wurde zusätzlich die Effektstärke (d) berechnet [Lipsey und Wilson, 2000]. Werte bis 0,5 geben eine kleine, bis 0,8 eine mittlere und darüber eine große bis sehr große Effektstärke an [Cohen, 1992].

\section{Anwendbarkeit der Intervention - Ergebnisse der Analysen}

\section{Subjektive Bewertung der Intervention}

Die Mehrheit der Interventionsteilnehmer gab eine unmittelbare positive Rückmeldung zur Intervention (Abb. 1a). Alle Teilnehmer fühlten sich verstanden, jeweils über $90 \%$ der Teilnehmer hielten die Intervention für Erfolg verspre- 
chend, würden sie weiter empfehlen und plädierten dafür, solche Interventionen häufiger anzubieten.

Zum Erhebungszeitpunkt 4 Wochen nach der Intervention gab die Mehrzahl der Teilnehmer an, sich mindestens eine der in der Intervention besprochenen Bewältigungsstrategien zur Umsetzung im Alltag vorgenommen zu haben (Abb. 1b). Die Beurteilung der Wirksamkeit der erprobten Bewältigungsstrategien fiel ebenfalls überwiegend positiv aus (Abb. 1c). Etwa $60-70 \%$ der Teilnehmer gaben an, einen positiven Einfluss auf ihr Befinden bemerkt und einer Verschlechterung des Befindens vorgebeugt zu haben. Dennoch gaben etwa $35 \%$ an, keinen Einfluss auf ihr Befinden bemerkt zu haben.

\section{Erreichbarkeit: Analyse der Refuser}

Als weiteres Maß der Anwendbarkeit der Intervention wurden Anteil und Charakteristika der Refuser analysiert. Von 248 zur Studienteilnahme geeigneten Patienten konnten aufgrund organisatorisch-struktureller Gründe 4\% (n = 9) nicht für die Interventionsstudie rekrutiert werden. Weitere $42 \%(n=103)$ lehnten eine Teilnahme ab, diese Personen stellen die Gruppe der Refuser dar. Die am häufigsten genannten Gründe für die Ablehnung waren zu großer zeitlicher oder logistischer Aufwand (46\% aller Refuser, $n=47$ ) und kein Interesse an einer Studienteilnahme $(30 \%, \mathrm{n}=31)$. Ein Fünftel der Refuser $(20 \%, n=21)$ fühlte sich durch das Konzept der Intervention nicht angesprochen und hielt eine Teilnahme nicht für notwendig. Weitere $4 \%(n=4)$ waren skeptisch bezüglich der Fragebögen bzw. der Studie.

Der Vergleich von Refusern und den Patienten, die randomisiert an der Interventionsstudie teilnahmen zeigte, dass die Interventionsgruppe im Kontrast zu den Refusern zum Erhebungszeitpunkt vor der Kardiodiagnostik signifikant jünger war $(d=0,46)$ sowie ungünstigere Krankheitsüberzeugungen ( $d=0,34-0,46)$ und eine höhere maximale Brustschmerzintensität $(\mathrm{d}=0,41)$ aufwies (Tab. 2). Patienten mit insignifikanten Einengungen der Koronargefäße lehnten eine Teilnahme an der Intervention signifikant häufiger ab als Patienten mit glattwandigen Koronargefäßen (Effektstärke d =0,57).

\section{Diskussion}

Unter den vielfältigen Missempfindungen im Brustbereich, die Patienten in medizinischen Kontexten berichten, sind schmerzbezogene Empfindungen häufig beobachtbar und bleiben nicht selten ohne kardiologisches Korrelat. Dieser sogenannte nicht-kardiale Brustschmerz nimmt oft einen chronischen Verlauf, auch wenn ernsthafte kardiale (und häufig generell somatische) Ursachen ausgeschlossen werden können. Jonsbu et al. [2012] berichteten einen ungünstigen Verlauf subjektiver Krankheitsannahmen bei Brustschmerzpatienten nach einfacher ärztlicher Rückmeldung unauffälliger Befunde. Dabei ist zu berücksichtigen, dass ein ungünstiger Beschwerdeverlauf auch iatrogen bedingt sein kann, bei- spielsweise aufgrund unvollständiger, verängstigender oder stigmatisierender Befundmitteilung ohne Einbezug der subjektiven Krankheitsannahmen der Patienten (im Sinne eines Nocebo-Effektes, Rief et al. [2008] und Schaefert et al. [2012]). Es wird daher gefordert, Interventionen anzubieten, die auf ein besseres Verständnis und eine bessere persönliche Kontrolle der Beschwerden abzielen [Jonsbu et al., 2012]. In Übereinstimmung mit dieser Forderung war das Ziel der hier vorgestellten verhaltensmedizinischen Intervention, den Betroffenen ein multifaktorielles Erklärungsmodell sowie Bewältigungsmöglichkeiten der Beschwerden zu vermitteln, um so einer Chronifizierung der Beschwerden entgegenzuwirken. Unsere Analysen zur Anwendbarkeit der Intervention zeigten eine überwiegend positive Bewertung der Intervention durch die Brustschmerzpatienten, wenngleich ein nicht $\mathrm{zu}$ vernachlässigender Teil der betroffenen Zielgruppe nicht erreicht werden konnte.

Insbesondere die unmittelbaren Rückmeldungen zur Intervention waren fast ausschließlich positiv. Dass sich alle Interventionsteilnehmer in ihrer Situation verstanden fühlten, verdeutlicht die Bedeutung der Kommunikation für den Umgang mit dieser Patientengruppe. Der von den meisten Patienten geäußerte Wunsch danach, solche Interventionen häufiger anzubieten, weist darauf hin, dass die routinemäßige Rückmeldung unauffälliger Befunde in ärztlichen Settings bislang als nicht befriedigend erlebt wird. Bislang kommt Patienten mit auffälligen Befunden in der Regel deutlich mehr Beratung zu als Patienten mit unauffälligen Untersuchungsbefunden [Nijher et al., 2001]. Im Unterschied zu Patienten mit auffälligen Befunden mangelt es Personen mit nicht-kardialen Brustschmerzen sowie Personen mit anderen unspezifischen Missempfindungen jedoch häufig an Erklärungs- und Beeinflussungsmöglichkeiten der subjektiv belastenden Beschwerden, sodass gerade diese Personengruppe mehr Aufmerksamkeit benötigt. Der hohe Prozentsatz an Interventionsteilnehmern, die mindestens eine der besprochenen Bewältigungsstrategien im Alltag angewandt hatten, kann ein Hinweis dafür sein, dass es sich lohnt, auch bei Patienten mit unauffälligen somatischen Befunden mehr Zeit in die Befundrückmeldung zu investieren.

Dennoch darf auch die Erreichbarkeit der betroffenen Zielgruppe als ein weiteres Maß der Anwendbarkeit der Intervention nicht außer Acht gelassen werden. Wir stellten eine insgesamt relativ hohe Rate an Patienten fest, die zur Studienteilnahme geeignet waren, aber einer Teilnahme nicht zustimmten. Gründe für eine Ablehnung psychologischer Interventionsangebote bei unspezifischen Brustsensationen können vielfältig sein, und auch unsere Stichprobe gab verschiedenste Gründe an. Bei einem Drittel bestand kein Interesse an einer Teilnahme; die Beweggründe dafür können an dieser Stelle nur vermutet werden. Optimalerweise fühlten sich diese Personen bereits aufgrund der ärztlichen Rückmeldung über unauffällige Befunde hinreichend beruhigt. Fraglich bleibt jedoch der weitere Verlauf dieser subjektiven 
Beruhigtheit. Bekannt ist, dass die anfängliche Beruhigtheit in Interaktion mit fortbestehenden Beschwerden und deren Interpretation zunehmend nachlässt [Donkin et al., 2006]. Es mögen weitere Motive existieren, die zu einer Ablehnung der Teilnahme aufgrund mangelndem Interesse geführt haben. Möglicherweise lehnte ein Teil dieser Personengruppe einen psychologisch geprägten Zugang zu den Beschwerden aufgrund eines stark somatischen Krankheitsverständnisses oder aufgrund genereller Vorurteile gegenüber «psychologischen Gesprächen» ab. Auch Prozesse der Vermeidung unangenehmer Gefühle und Gedanken (z.B. bezüglich der Bedrohlichkeit der erlebten Beschwerden) könnten hier eine Rolle gespielt haben. Jedoch gaben nur wenige Personen explizit an, sich durch das Konzept der Intervention nicht angesprochen zu fühlen. Dieses Ergebnis lässt sich in Bezug auf das Konzept der «Pathologischen Realangst» [Linden et al., 2008] diskutieren, nach dem körperliche Beschwerden nicht zwangsläufig für die Betroffenen problematisch sind, sondern erst durch eine pathologische Verarbeitung interventionsbedürftig werden. Damit übereinstimmend fanden wir bei den Interventionsteilnehmern nicht nur eine stärkere emotionale Beeinträchtigung, sondern auch ungünstigere subjektive Annahmen bezüglich ihrer Brustschmerzen als bei den Nichtteilnehmern (z.B. hinsichtlich der erwarteten Folgen).

In der vorgestellten Studie wurden formal-organisatorische Aspekte (z.B. fehlende Zeit, Reiseaufwand) am häufigsten zur Begründung einer Nichtteilnahme angeführt. Entsprechend sollten Überlegungen angestellt werden, wie Interventionen dieser Art besser in die Routineversorgung integriert werden können. Auch vorherige Studien [Mayou et al., 1999; Sanders et al., 1997] kommen zu der Schlussfolgerung, dass den Betroffenen ein leichterer und unkomplizierter Zugang $\mathrm{zu}$ psychologischen Interventionen ermöglicht werden muss. Die Tatsache, dass in unserer Studie im Mittel etwa 2 Wochen vergingen, bis ein Termin zur Teilnahme an der Intervention gefunden wurde, zeigt, dass es selbst für die Patienten mit Interesse an der Intervention nicht einfach war, dieses zusätzliche Angebot in Anspruch zu nehmen. Eine stärkere Verankerung in die Versorgungsstruktur könnte vermutlich erreicht werden, indem die Intervention direkt in Verbindung mit dem Termin zur kardiologischen Diagnostik eingeplant und als ausführliches Rückmeldegespräch angeboten wird (ohne separate Terminvereinbarung). Eine Alternative wäre, niederschwellige Telefon- oder E-Mail-Kontakte anzubieten oder die wichtigsten Informationen in einem Faltblatt bereitzustellen [Arnold et al., 2009].

Die hier vorgestellte Intervention zielte ausgehend von subjektiven Krankheitskonzepten der Betroffenen auf entsprechende Bewältigungsstrategien bezüglich der erlebten Missempfindungen im Brustbereich ab. Unsere Ergebnisse zeigen insgesamt, dass diese niederschwellige Intervention von Patienten mit nicht-kardialem Brustschmerz überwiegend positiv bewertet wurde. Eine ungünstige psychische Verarbeitung von somatischen Beschwerden ist jedoch auch bei Perso- nen mit zugrundeliegender somatischer Pathologie vorzufinden, die somit ebenfalls von ähnlichen psychologischen Interventionen profitieren könnten. Auch die Veränderungen der Diagnosekriterien für die «somatic symptom disorder» im DSM-5 fokussieren nun mehr auf Prozesse der psychologischen Verarbeitung von Beschwerden als auf ein zugrundeliegendes organpathologisches Korrelat [American Psychiatric Association, 2013]. Eine ängstliche Bewertung von Missempfindungen im Brustbereich, übermäßige Konzentration auf das Herz, ausgeprägte Schonhaltung und Vermeidung von symptomverstärkenden Aktivitäten gelten generell als Indikatoren eines komplizierten Krankheitsverlaufs [Linden et al., 2008; Schaefert et al., 2012]. Der deutliche Vorteil von entsprechend ausgerichteten niederschwelligen Interventionen im Rahmen einer gestuften Versorgung liegt in der relativ guten Integrierbarkeit in die Strukturen der primär- oder sekundärmedizinischen Versorgung. Auch in der aktuellen S3-Leitlinie «Brustschmerz» der Deutschen Gesellschaft für Allgemein- und Familienmedizin (DEGAM) wird Behandlern in diesen Versorgungssettings empfohlen, psychosoziale Begleitsymptome bei Patienten mit Brustschmerz zu erfragen [DEGAM, 2011]. Das Potenzial der Berücksichtigung psychosozialer Aspekte bei Brustschmerz und anderen Missempfindungen im Brustbereich zeigt sich in der Verhinderung einer Chronifizierung der Beschwerden und assoziierter Beeinträchtigungen. Um dieses Angebot für viele Betroffene erreichbar zu machen, sollten Interventionen optimalerweise als fester Bestandteil in die Routineversorgung integriert werden. Wirksamkeitsanalysen dieser Intervention im Vergleich zu einer routinemäßig versorgten Kontrollgruppe stehen noch aus.

\section{Dank}

Wir danken Prof. Dr. M. de Zwaan (Klinik für Psychosomatik und Psychotherapie, Medizinische Hochschule Hannover), Prof. Dr. S. Achenbach, Prof. Dr. W. G. Daniel (Medizinische Klinik 2, Universitätsklinikum Erlangen) und PD Dr. F.-K. Pohle (Medizinische Klinik 1, Krankenhaus Martha-Maria Nürnberg) für die freundliche Unterstützung im Verlauf des Projekts.

\section{Disclosure Statement}

Die Studie wurde gefördert durch den ELAN-Fonds (Erlanger Leistungsbezogene Anschubfinanzierung und Nachwuchsförderung) der Medizinischen Fakultät der Friedrich-Alexander-Universität ErlangenNürnberg. Es bestehen keine Interessenskonflikte. 


\section{Literatur}

Arnold J, Goodacre S, Bath P, Price J: Information sheets for patients with acute chest pain: Randomised controlled trial. BMJ 2009;338:b541.

American Psychiatric Association: Diagnostic and Statistical Manual of Mental Disorders, Fifth Edition. Washington D.C., American Psychiatric Publishing, 2013.

Bass C, Mayou R: Chest pain. BMJ 2002;325:588-591.

Broadbent E, Petrie KJ, Main J, Weinman J: The brie illness perception questionnaire. J Psychosom Res 2006;60:631-637.

Cohen J: A power primer. Psychol Bull 1992;112:155159.

Deutsche Gesellschaft für Allgemein- und Familienmedizin (DEGAM): DEGAM S3-Leitlinie Nr. 15 «Brustschmerz». Düsseldorf, Omikron Publishing, 2011.

Deutsche Schmerzgesellschaft: Deutscher Schmerzfragebogen. www.dgss.org/deutscher-schmerzfragebogen (Zugriff am 21.10.2013).

Donkin L, Ellis CJ, Powell R, Broadbent E, Gamble $\mathrm{G}$, Petrie KJ: Illness perceptions predict reassurance following a negative exercise stress testing result. Psychol Health 2006;21:421-430.

-Esler JL, Barlow DH, Woolard RH, Nicholson RA, Nash JM, Erogul MH: A brief cognitive-behavioral intervention for patients with noncardiac chest pain. Behav Ther 2003;34:129-148.

Eslick GD, Fass R: Noncardiac chest pain: evaluation and treatment. Gastroenterol Clin North Am 2003, 32:531-552.

Glombiewski J, Rief W, Bösner S, Keller H, Martin A Donner-Banzhoff N: The course of nonspecific chest pain in primary care: symptom persistence and health care usage. Arch Intern Med 2010; 170:251-255.

- Jonsbu E, Martinsen EW, Morken G, Moum T, Dammen T: Illness perception among patients with chest pain and palpitations before and after negative cardiac evaluation. Biopsychosoc Med 2012;6 19-31.

Kahn SE: The challenge of evaluating the patient with chest pain. Arch Pathol Lab Med 2000;124:1418 1419.
Krille S, Martin A: Gesundheitsangst bei funktionellen Herzbeschwerden; in Hoefert W, Klotter C (eds): Gesundheitsängste. Lengerich, Pabst Science Publishers, 2011, pp 130-145.

Kroenke K, Arrington ME, Mangelsdorff AD: The prevalence of symptoms in medical outpatients and the adequacy of therapy. Arch Intern Med 1990; 150:1685-1689.

Kroenke K, Price RK: Symptoms in the community. Prevalence, classification, and psychiatric comorbidity. Arch Intern Med 1993;153:2474-2480.

Leventhal H, Leventhal EA, Contrada RJ: Self-regulation, health, and behaviour: a perceptual-cognitive approach. Psychol Health 1998;13:717-733.

Linden M, Dirks S, Glatz J: Die «Pathologische Realangst» am Beispiel kardiovaskulärer Erkrankungen. Psychosomatik und Konsiliarpsychiatrie 2008; 2:248-254.

Lipsey MW, Wilson DB: Practical Meta-Analysis. Thousand Oaks, Sage Publications, 2000.

Martin A, Rauh E, Fichter M, Rief W: A one-session treatment for patients suffering from medically unexplained symptoms in primary care - a randomized clinical trial. Psychosomatics 2007;48:294-303.

Mayou R: Invited review: atypical chest pain. J Psychosom Res 1989;33:393-406.

Mayou R, Farmer A: Functional somatic symptoms and syndromes. BMJ 2002;325:265-268.

Mayou RA, Bass CM, Bryant BM: Management of non-cardiac chest pain: from research to clinical practice. Heart 1999;81:387-392.

Nanke A, Rief W: Biofeedback-Therapie bei somatoformen Störungen. Verhaltenstherapie 2000;10: 238-248.

Nijher G, Weinman J, Bass C, Chambers J: Chest pain in people with normal coronary anatomy. BMJ 2001:323:1319-1320.

Papanicolaou MN, Califf RM, Hlatky MA, McKinnis RA, Harrell FE, Jr., Mark DB, McCants B, Rosati RA, Lee KL, Pryor DB: Prognostic implications of angiographically normal and insignificantly narrowed coronary arteries. Am J Cardiol 1986;58: 1181-1187.
Potts SG, Bass CM: Psychosocial outcome and use of medical resources in patients with chest pain and normal or near-normal coronary arteries: a longterm follow-up study. Q J Med 1993;86:583-593.

Rief W, Hiller W: Somatisierungsstörung und Hypochondrie. Göttingen, Hogrefe, 1998

Rief W, Nestoriuc AY, Hofmann SG: The power of expectation - understanding the placebo and nocebo phenomenon. Social and Personality Psychology Compass 2008;2:1624-1637.

Robertson N, Javed N, Samani NJ, Khunti K: Psychological morbidity and illness appraisals of patients with cardiac and non-cardiac chest pain attending a rapid access chest pain clinic: a longitudinal cohort study. Heart 2008;94:e12.

Sanders D, Bass C, Mayou RA, Goodwin S, Bryant BM, Tyndel S: Non-cardiac chest pain: why was a brief intervention apparently ineffective? Psychol Med 1997;27:1033-1040.

Schaefert R, Hausteiner-Wiehle C, Häuser W, Ronel J, Herrmann M, Henningsen P: Clinical practice guideline: non-specific, functional and somatoform bodily complaints. Dtsch Arztebl Int 2012;109:803813.

Schappert SM: National ambulatory medical care survey: 1989 summary. Vital and health statistics Series 13. Data from the National Health Survey 1992: $1-80$

Schmulson MJ, Ortiz-Garrido OM, Hinojosa C, Arcila D: A single session of reassurance can acutely improve the self-perception of impairment in patients with ibs. J Psychosom Res 2006;61:461-467.

Zimmermann K, Einsle F, Dannemann S, Pollack K, Kollner V, Nitschke-Bertaud M: Psychische und physische Befindlichkeit von Patienten mit Thoraxschmerzen in der kardiologischen Ambulanz. Psychother Psych Med 2010;60:368-378. 